\title{
Research on Petrol Secondary Distribution with Stochastic Demand
}

\author{
Pan Cen, Can Cong, Zhenping Li \\ Information Institute, Beijing Wuzi University, Beijing, China
}

Email address:

1742873403@qq.com (Pan Cen)

\section{To cite this article:}

Pan Cen, Can Cong, Zhenping Li. Research on Petrol Secondary Distribution with Stochastic Demand. Science Journal of Energy Engineering. Vol. 4, No. 4, 2016, pp. 30-34. doi: 10.11648/j.sjee.20160404.11

Received: October 10, 2016; Accepted: October 20, 2016; Published: November 14, 2016

\begin{abstract}
Petrol is a kind of strategic natural resources. Providing legitimate transportation plans for the petrol secondary distribution is the key links to guarantee the petrol normal sales. The aim of this paper mainly is to obtain a distribution plan to meet certain level of service considering stochastic demand circumstances. Factors including the sales volume, the initial inventory, different type vehicles and capacity limitation constraints are considered. Firstly, a mathematical model for minimizing the total cost of petrol secondary distribution is built on the premise of considering various factors. Then a hybrid algorithm is designed by combining the greedy algorithm and the saving algorithm. The greedy algorithm is used to find a local optimal solution, and the saving algorithm is used to adjust the solution. Finally, the hybrid algorithm is used to solve a specific cases, which verifying the feasibility of the algorithm.
\end{abstract}

Keywords: Petrol Secondary Distribution, Mathematical Model, The Greedy Algorithm, The Saving Algorithm

\section{Introduction}

Vendor Managed Inventory strategy with systematic and integrated inventory management thought effectively solves the bullwhip effect, breaking the traditional fragmented inventory management model. IRP (Inventory Routing Problem) become a new research field at the driven on two realities demand of how to optimize logistics chain of distribution and inventory, which is core issues in the process of the implementation of VMI strategy. IRP is widely exists in many industries such as petroleum chemical industry, automobile manufacturing, supermarket chains, household items, etc. At the same time, an effective solution to this problem can save considerable cost for the enterprise.

Opening China's oil market making an increasingly competitive market, coupled with the scarcity of oil resources, and services requirements increasing, state-owned marketing enterprises are facing tremendous pressure. Designing a reasonable petrol secondary distribution is a core problem of oil marketing enterprises effectively integrating enterprise logistics by integrating enterprise resources, which play a very crucial role in promoting the overall logistics cost reduction.

Petrol distribution can be divided into primary distribution and secondary distribution. The process of petrol transportation from refineries to depots is called primary distribution and the process of petrol transportation from depots to petrol stations or other terminal customers is called secondary distribution. The main participants of petrol secondary distribution are dispatching center, depots, carriers and petrol stations. As shown in Figure 1, dispatching center forecasts the replenishment quantity every day based on the sales data received from petrol stations, then base on the information of depots and vehicles, the dispatching plan is generated which would be executed by carriers [1].

Many papers study the oil distribution problems under certain demand, but relatively few study about stochastic demand. $\mathrm{Li}$ [2] had put forward the basic model of oil logistics distribution, and proposed integrated logistics solutions, made a prediction model for the oil distribution, according to vendor-managed inventory theory, and finally proved that the model has optimal results. Xu [3] analyzed the actual situation of transport oil, and he used genetic algorithm to conduct a comprehensive analysis, while adding time window constraints. Li [4] designed the refined oil distribution model and used the improved genetic algorithm to solve it, which optimize the logistics processes by adding a number of auxiliary calculation method to improve the overall solution process. Ma [5] raised a 
mathematical model of the secondary distribution of refined oil, focusing on the genetic algorithm and using $\mathrm{C}$ language to program, finding the advantages of using a genetic algorithm. Although the number of papers studying oil distribution problems with stochastic demand is few, the relevant study still exists. Solving secondary distribution problems can based on these papers. Bard and Nananukul [6] proposed how to arrange distribution plan for the uncertainty customer demand study under the certain plan period, specific to the logistics system about a single supplier service for multiple customer. From the angle of the inventory and transportation costs minimally, it determined who need to service, and used the heuristic algorithm to solve the mixed integer programming model. Dong Won Cho [7] Studies inventory routing problem based on the one time cycle, and designed a kind of adaptive genetic algorithm to solve problems. Adelman[8] discussed the problem under the uncertain demand. The problem is under the guidance of price, and each customer's demand obeys markov process, so that the author used the two-stage method to solve the optimal problem. Mohd [9] built the logistics distribution model, considering the influence of the service level. He used Lagrange heuristic algorithm to solve the problem, and also designed the heuristic algorithm to optimize the initial solution. Zhao [10] analyzed the composition of optimal strategy when the customer demand is independent identically distributed, and designed a algorithm to verify the feasibility. The paper also studied problem considering time, including soft time window and hard time window. Qureshi et al [11] proposed that the type of time window can be classify to three according time constraints. They are the hard time window not allowed to wait, the hard time window allowed to wait, and the soft time windows.

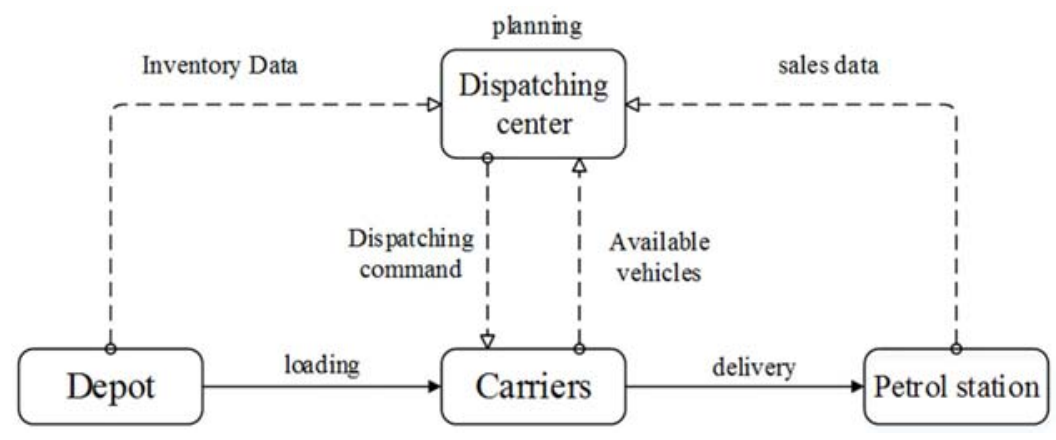

Figure 1. Procedure of secondary distribution.

In this paper, we research on the problem of the petrol secondary distribution strategy when the sales volume of each station obeys normal distribution. In order to simplify the problem, shortage cost directly is not considered, but the service level reflects the shortage of loss. That is to say, when meeting a certain service level, shortage of losses is not considered. In this case, the main factor affecting the total cost of shipping is the unit cost of vehicle transportation, transportation distance and vehicle number, namely the transportation cost.

\section{Mathematical Model of the Petrol Secondary Distribution Problem with Stochastic Demand}

\subsection{Problem Description}

Assume that there are $\mathrm{n}$ petrol stations in an area whose petrol is delivered from one depot. Assume that the petrol must be delivered from the depot to petrol stations by full loaded compartment vehicles, and the petrol in one compartment must be fully unloaded into one oil tank rather than separated to multiple oil tanks. There are $k$ kinds of vehicles whose compartments capacity are known, each kind of vehicles are sufficient. The capacity of each station's oil tank, the unit transportation cost from the depot to every station and initial inventory are known. Sales volume of each station is normal distribution, and independent of each other. The question is how to arrange the transportation plan so as to minimize the total cost under the premise of the service level.

\subsection{Mixed integer Programming Model}

To establish the mathematical model of the problem, some symbols and variables are defined as follows.

$M=\{1,2, \ldots, n\}:$ a collection of petrol station;

$M^{\prime}=\{0,1,2, \ldots, n\}:$ a collection of petrol station and depot;

$r=\{1,2, \ldots, k\}:$ a collection of vehicle;

$t=\{1,2, \ldots, T\}:$ a collection of time;

$c_{r}:$ unit transportation cost of vehicle r;

$Q_{r}$ : compartment capacity of vehicle type r;

$I_{t i}$ : the inventory volume of petrol station at time t;

$d_{i t}$ : the average of sales volume;

$\delta_{i}$ : standard deviation of sales volume;

$H_{i}$ : the upper bounds of each station's oil tank;

$u_{i j}$ : distance from station $\mathrm{i}$ to station $\mathrm{j}$;

$x_{t i j r}$ : a binary variable set to 1 if station $\mathrm{j}$ is visited immediately after station $i$ by vehicle $r$ in period $t$, and 0 otherwise;

$y_{\text {tir }}$ : a binary variable set to 1 if vehicle $r$ is being used in time $\mathrm{t}$, and 0 otherwise; 
The petrol secondary distribution problem can be formulated into the following mixed integer programming model:

$$
\begin{gathered}
\min z=\sum_{t=0}^{T} \sum_{i=0}^{n} \sum_{j=0}^{n} \sum_{r=1}^{k} c_{r} x_{t i j r} u_{i j} \\
\sum_{i=0}^{n} x_{t i m r}=\sum_{j=0}^{n} x_{t m j r} . \forall t \in T, \forall m \in M, \forall r \in k \\
\sum_{i=0}^{n} \sum_{r=1}^{k} x_{t i m r}=\sum_{j=0}^{n} \sum_{r=1}^{k} x_{t m j r}, \forall t \in T, \forall m \in M \\
\sum_{r=1}^{k} Q_{r} y_{t i r}+I_{i t} \leq H_{i}, \forall i \in M, \forall t \in T \\
I_{i t}+W_{i(t+1)} \geq d_{i}+z_{\alpha} \delta_{i}, \forall i \in M, \forall t \in T \\
W_{i t}=\sum_{r=1}^{k} Q_{r} y_{t i r}, \forall i \in M, \forall t \in T \\
x_{t i j r}, y_{t i r} \in\{0,1\} \quad \forall t \in T, \forall i, j \in n, \forall m \in M, \forall r \in k \\
I_{t i}, Q_{r}, d_{i} \geq 0 \quad \forall t \in T, \forall i \in n, \forall r \in k
\end{gathered}
$$

Object function (1) minimizes the total cost of the petrol secondary distribution. Constrains (2) assure that if a vehicle arrives at a station, it must leave after if has served it to a next station or to the depot. Constrains (3) assure that the number of vehicle arriving a station must equal to the number of vehicle leaving. Constrains (4) assure that the quantity delivered to a retailer add inventory cannot excess the station capacity. Constrains (5) prevent stockout from occurring at each retailer with a confidence level $\alpha$ during each period of the planning horizon. A service level of $\alpha$ is defined by the standard normal value $Z_{\alpha}$. Constrains (6) denote the quantity delivered to a station at time t. Constrains (7) and (8) are the illustrations of variables.

\section{Solving Method}

In the study of petrol secondary distribution optimization, it is difficult to get optimal solution by solving the model directly in a short time. Because it contains a wide variety of elements, variables and constraints of abstract general model, and also include integer variable. Therefore, designing a hybrid algorithm for this problem is needed. At first, an initial solution is achieved using the greedy algorithm and then optimize the initial solution by the saving algorithm.

\subsection{Finding a Initial Solution by the Greedy Algorithm}

The greedy algorithm steps as the following:

(1) Input the initial inventory of petrol stations, the mean of sales volume at unit time, standard deviation, vehicle starting time, beginning time of petrol station service, ending time, $Q_{r}, I_{t i}, d_{i t}, H_{i}, u_{i j}$
(2) Calculate the distribution cost of each type vehicle, rank the distribution cost in an ascending sort order, according to the unit cost $c_{1}, c_{2}, \ldots, c_{k}, c_{k}=\frac{C_{k}}{Q_{k} * u}, C_{k}$ is transportation cost of vehicle $\mathrm{k}$;

(3) Calculate stock time series of the petrol stations in the first round and sort by successively $t_{1}, t_{2}, \ldots, t_{n}, t=\frac{I_{i t}}{q_{i t}}$, the sales volume of stations measured by mean;

(4) Arrange vehicle of lower cost to stations which meet constraint; When it come to several bin, transportation time are supposed to considered in stock time series. Delivery quantity can be calculated according to $I_{i t}+W_{i(t+1)} \geq d_{i}+z_{\alpha} \delta_{i}$ Regarding the lowest value as forecast volume of sales;

(5)Update $t_{1}$ and the stations inventory according to distribution plan;

(6) Calculate the stock time series in the next round $t_{1}^{\prime}, t_{2}^{\prime}, \ldots, t_{n}^{\prime}$, as stations ending inventory will change after finishing distribution plan. Ending inventory can be calculated according to, $I_{i t+1}=I_{i t}+W_{i t}-d_{i t}, W_{i(t+1)}$ is depended on $z_{\alpha}$ and $\delta_{i}$. Use the lowest value of $W_{i(t+1)}$ as delivery quality according step (4). New time series can be obtained by method same as step (3), and ensure which station to distribute;

(7) If $t_{1}^{\prime}$ exceed or equal to ending time, the algorithm stop, else back to step (3);

(8) Output the distribution plan of all stations.

\subsection{Optimizing the Initial Solution by the Saving Algorithm}

Although the approximate solution obtained by the greedy choice is not necessarily the optimal solution, it can optimize the initial solution from several aspects by the saving algorithm on the basis of the approximate solution, making it close to the optimal solution.

The saving algorithm steps are as follows:

(1) Order all vehicle loading capacity in an ascending order $w_{1}, w_{2}, \ldots, w_{k}$, the corresponding cost are $c_{1}, c_{2}, \ldots, c_{k}$;

(2) If the ending inventory exceed or equal to $w_{x}-w_{y}$, $w_{m}-w_{j} \ldots \ldots, c_{x}>c_{y}, c_{m}>c_{j} \ldots \ldots$, and the saving cost is maximum, the vehicle $\mathrm{x}$ can be replaced by the vehicle $\mathrm{y}$;

(3) If a station ending inventory exceed or equal to $w_{x}-w_{y}$ and $c_{x}>c_{y}$, search time window sequences using the forward way, the vehicle $\mathrm{x}$ can be replaced by the vehicle $\mathrm{y}$;

(4) In the condition of not just one bin, it will cause correspond delivery quantity excess, which can also solve by the ideas of optimization above;

(5) Use the greedy algorithm to get the distribution plan after optimizing the time window sequences;

(6) If the ending inventory still can be optimized, use the saving algorithm again until there have no space to optimize.

The process of the hybrid algorithm combining the greedy algorithm and the saving algorithm solve IRP is described as figure 2 . 


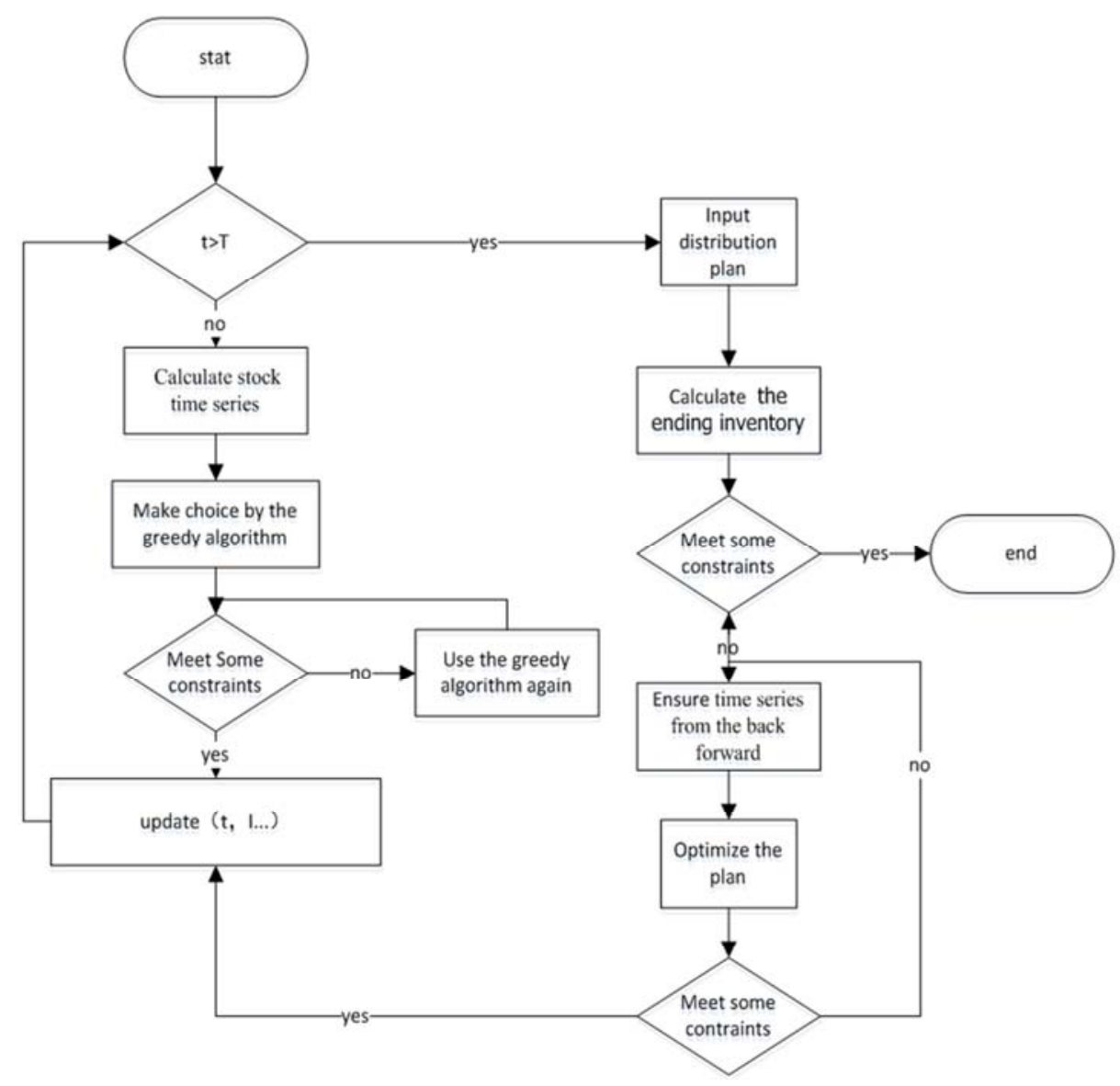

Figure 2. The hybrid algorithm process.

\section{Example Analysis}

\subsection{Example Description}

A region has a depot distributing for five stations. As the distribution plan is based on experience at the past, causing high cost, dispatching center decide to rearrange distribution plan. In order to ensure that all stations meet a certain service level, the plan dispatcher need arrange the delivery plan according to the inventories of oil tank every morning. Information of petrol stations, oil tank and vehicle can are presented in the table 1,2 . Sales volume of each station obeys normal distribution and is independent of each other. The mean of normal distribution is equal to the average sales volume per hour of petrol station in the below table 1, and the standard deviation is 100 .

Table 1. Information of petrol stations and oil tank.

\begin{tabular}{llll}
\hline \multirow{2}{*}{$\begin{array}{l}\text { Petrol } \\
\text { station }\end{array}$} & \multicolumn{3}{l}{ Oil tank information (unit with $\mathbf{L}$ ) } \\
\cline { 2 - 4 } & $\begin{array}{l}\text { capacity of oil } \\
\text { tank }\end{array}$ & $\begin{array}{l}\text { Average of } \\
\text { sales volume }\end{array}$ & $\begin{array}{l}\text { Initial inventory } \\
\text { at 8:00 AM }\end{array}$ \\
\hline S1 & 17000 & 2000 & 8010 \\
S2 & 17000 & 3000 & 6008 \\
S3 & 19000 & 5000 & 15015 \\
S4 & 40000 & 8000 & 16020 \\
S5 & 26500 & 4000 & 26009 \\
\hline
\end{tabular}

Table 2. Vehicle information.

\begin{tabular}{llll}
\hline Vehicle type & capacity & The freight one time \\
\hline One bin & $8000 \mathrm{~L}$ & & $100 \mathrm{RMB}$ \\
Two bin & $10000 \mathrm{~L}$ & 10000L & $120 \mathrm{RMB}$ \\
\hline
\end{tabular}

\subsection{Solve Process}

$s_{1}$ : denotes using the first type vehicle to service stations, and the distribution volume is 8000 ;

$s_{2}$ : denotes using the second type vehicle to service stations, the distribution volume is 10000 ;

$s_{3}$ : denotes using the second type vehicle to service stations, the distribution volume is 20000 .

Comparing the distribution cost, $s_{3}$ is less, $s_{2}$ next, and $s_{1}$ most. Therefore, adopt $s_{3}$ first, $s_{2}$ next, and $s_{1}$ last. The best strategy is chose by the lowest cost. $s_{p}(i, j)$ denotes service station $\mathrm{i}$ and then $\mathrm{j}$ with strategy $s_{p}$

1. The initial solution obtained by the greedy algorithm

Table 3. Initial solution

\begin{tabular}{lllllll}
\hline $\mathbf{9 : 5 3}$ & $\mathbf{1 0 : 5 4}$ & $\mathbf{1 1 : 4 1}$ & $\mathbf{1 2 : 5 0}$ & $\mathbf{1 3 : 3 7}$ & $\mathbf{1 4 : 4 6}$ & $\mathbf{1 6 : 0 0}$ \\
\hline$s_{2}(2,4)$ & $s_{2}(3,4)$ & $s_{2}(1,4)$ & $s_{2}(2,3)$ & $s_{2}(4,5)$ & $s_{2}(3,4)$ & $s_{2}(2,4)$ \\
$16: 31$ & $16: 42$ & $18: 31$ & $19: 00$ & $19: 45$ & $20: 55$ & $21: 26$ \\
$s_{2}(1,5)$ & $s_{2}(3,4)$ & $s_{2}(3,4)$ & $s_{2}(2,5)$ & $s_{2}(3,4)$ & $s_{2}(1,4)$ & $s_{2}(4,5)$ \\
\hline
\end{tabular}


According to table 3, the distribution strategy is showed in detail. The second type vehicle deliver 10000 to station 2 at 9:53 firstly, then deliver 10000 to station 4; The second type vehicle deliver 10000 to station 3 at 10:54 firstly, then deliver 10000 to station 4 ; the distribution plan at other time and so on.

The final inventory as following:

$$
I_{1}=7700, I_{2}=1698, I_{3}=2705, I_{4}=11710, I_{5}=7699 .
$$

The total cost $\mathrm{c}=1680$.

2. Optimizing the initial solution

The final optimal solution can be obtained as following:

Table 4. Adjust solution.

\begin{tabular}{lllllll}
\hline $\mathbf{9 : 5 3}$ & $\mathbf{1 0 : 5 4}$ & $\mathbf{1 1 : 4 1}$ & $\mathbf{1 2 : 5 0}$ & $\mathbf{1 3 : 3 7}$ & $\mathbf{1 4 : 4 6}$ & $\mathbf{1 6 : 0 0}$ \\
\hline$s_{2}(2,4)$ & $s_{2}(3,4)$ & $s_{2}(1,4)$ & $s_{2}(2,3)$ & $s_{2}(4,5)$ & $s_{2}(3,4)$ & $s_{2}(2,4)$ \\
$16: 31$ & $16: 42$ & $18: 31$ & $19: 00$ & $19: 45$ & $20: 55$ & $21: 26$ \\
$s_{2}(1,5)$ & $s_{2}(3,4)$ & $s_{2}(3,4)$ & $s_{2}(2,5)$ & $s_{2}(3,4)$ & $s_{2}(1,4)$ & $s_{1}(5)$ \\
\hline
\end{tabular}

According to table 3 , the distribution strategy in detail is showed. The second type vehicle deliver 10000 to station 2 at 9:53 firstly, then deliver 10000 to station 4. By that analogy, the distribution plan at other time can be received. The delivery plan is same to the initial solution before $21: 26$, but at 21:26, Delivery 8000 to station 5 is enough.

The final inventory is as follows:

$$
I_{1}=7700, I_{2}=1698, I_{3}=2705, I_{4}=11710, I_{5}=5699 .
$$

The total cost $\mathrm{c}=1660$.

Using the saving algorithm on the basis of the initial solution saves 20 yuan after the adjustment. This example fully proved the feasibility of the algorithm. As the scale of the problem is small, saving cost is not so obvious. When it comes to a larger scale problem, the hybrid algorithm can still be applicable, and achieve the goal of reducing costs as far as possible.

\section{Conclusion}

Petrol secondary distribution optimization, not only can optimize transportation route, distribution structure, inventory, costs, gain huge profits to marketing company, but also save the delivery time, improve the service quality and increase consumer satisfaction. In the case of only considering the distribution cost, the paper discuses the problem of multiple time delivery. In this paper, the mathematical model of petrol secondary distribution is built after analyzing the various factors; and using the hybrid algorithm combining the greedy algorithm and the saving distribution to solve the problem can effectively receive an optimization solution; finally a specific example verify feasibility of the hybrid algorithm. Many scholars studied distribution problem with the determine demand, but the uncertain demand research is relatively few.

However customer demand mostly tend to be stochastic, it is necessary to do further research considering stochastic demand. To solve practical problems, although the problem description is not better than other intelligent algorithms, few scholars use the combination of the greedy algorithm and the saving algorithm in the IRP before. This Hybrid algorithm is a kind of innovation. But when dealing with the variety of practical problems, It needs adopt different skills to solve the problem effectively. For example, if there are several type vehicles with equal unit freight, replenishment quantity is decided by the petrol station sales per unit time, maximum capacity, and the relationship between them when using the greedy algorithm to get initial solution.

In practice, problem is far more complex than our model. In addition to the cost of transportation, the cost of storage, shortage, vehicle fixed cost, etc. also should be considered. And customer demand may obey other distribution or even no rules to follow. In the future, researching this kind of problem from the perspective of several costs and different demand distribution is our next task.

\section{References}

[1] Li Z., Jiang C. (2015) Study on the Transportation Problem of Petrol Secondary Distribution with Considering Shortage Cost. Open Journal of Modelling and Simulation, 4: 34-40.

[2] Li Q. Li S.(2008) A Novel Logistic Distribution Model of Refined Oil Based on Vendor or Managed Inventory. Journal of China University of Petroleum.

[3] Xu H. (2008) Research on The Optimization of Oil Field Transportation Vehicle Scheduling Based on Genetic Algorithm.

[4] Cong J. (2009) Research and Implementation of Oil Logistics Optimization. Xi'an University of Technology.

[5] Ma Y. (2010) Dispatching Optimization Model of Second Distribution of Gasolin \& Diesel Oil and Solution Based on Genetic Algorithm. Operations Research and Management Science, 19(06): 73-78.

[6] Bard J. F., Nananukul N. (2010) A Branch and Price Algorithm for An Integrated Producation and Dinventory Routing Problem [J]. Computers \& Operations Research, 37(22):02-17.

[7] Dong W., Young H., Tae Y., Mitsuo G. (2014) An adaptive genetic algorithm for the time dependent inventoryrouting problem [J], Journal of Intelligent Manufacturing, 25:10251042.

[8] Adelman Daniel Adelman. (2004) A Price-Directed Approach to Stochastic Inventory/Routing. Operation Reseach, 52:499-514.

[9] Mohd Kamarul Irwan Abdul Rahim,. Yi Q. (2014) Modelling and Solving The Multi-period Inventory Routing Problem With Stochastic Stationary Demand Rates [J]. International Journal of Production Research, 52(43)51-63.

[10] Zhao D. (2012) Research on Inventory Routing Problem With Stochastic Demand. Southwest Jiaotong University, 88-98.

[11] Qureshi A. G,. Taniguchi E, Yamada T. (2009) An Exact Solution Approach for Vehicle Routing and Scheduling Problem With Soft Time Windows. Transportation Research Part E, 45:960-977. 